\title{
Distributed Fusion Filter for Multi-rate Sampling Stochastic Singular Systems with Multiplicative Noises
}

\author{
Hao Jin ${ }^{1}$, Jing $\mathrm{Ma}^{2 *}$, Yun $\mathrm{Li}^{1}$ and Ming Zhao ${ }^{1}$ \\ ${ }^{1}$ School of Computer and Information Engineering, Harbin University of Commerce, \\ Harbin 150028, China \\ ${ }^{2}$ School of Mathematics Science, Heilongjiang University, Harbin 150080, China \\ majing427@gmail.com
}

\begin{abstract}
The distributed fusion filtering problem is studied for multi-rate sampling stochastic singular linear systems with multiple sensors and stochastic multiplicative noises. The system is described at the highest sampling rate and different sensors may have different lower sampling rates. The white noise in measurement matrix is introduced to describe the stochastic disturbance. Firstly, based on decomposition in canonical form, the original singular system is transformed into fast and slow two subsystems. For the two reduced-order subsystems, the local filters (LFs) are given based on the "dummy" random variables. The cross-covariance matrices between any two local filtering errors are derived. Further, the distributed fusion filter weighted by matrices (FFWM) is obtained for the original singular system based on the well-known fusion algorithm in the linear minimum variance sense. Simulation example verifies the correctness and feasibility of the proposed algorithm.
\end{abstract}

Keywords: Multi-rate, Distributed fusion, Singular system, Multiplicative noise, Filter

\section{Introduction}

In recent years, the information fusion filtering problem for systems with multiple sensors has gained lots of attention due to the widely applications such as target tracking, single processing and robot navigation [1].

When the stochastic system is measured by multiple sensors, there are two approaches to process the multiple measurements from different sensors. One is the centralized filter, the other is distributed fusion filter [2]. The centralized filter can give the global optimal estimation. However, it can result in high computational cost due to the high dimension augmented measurement. Recently, many researchers are focus on the distributed filter since it is easily for fault detection and isolation. There are many popular distributed fusion algorithms such as federated square-root filter [3], maximum likelihood fusion algorithm [4] and weighting fusion algorithms in the linear minimum variance sense [5]. However, the above algorithms are only suitable for single rate systems.

For multi-rate systems, the first important study goes back to the switch decomposition technique proposed by Kranc [6]. Generally, there are two methods for the state estimation problem for multi-rate systems. One is based on multiscale system theory and the other is based on Kalman filtering theory. On the basis of multiscale system theory, many famous fusion strategies are proposed for multirate systems with

${ }^{*}$ Corresponding Author: Jing Ma 
the sampling rate ratio being one or positive integer power to two. However, the state estimators are very complex and high computational burden. On the basis of Kalman filtering theory, many useful filtering strategies are proposed such as optimal signal reconstruction method [7], asynchronous centralized fusion algorithm [8], sequential filtering algorithm [9], and left synchronously lifting technology [10]. But, the computational cost of the above filtering strategies is high since they are given by state/measurement augmentation. In order to avoid the state augmentation, the multirate fusion problem is transformed into an equivalent single rate fusion problem. For non-uniform sampling systems, a distributed fusion filter is given [11], for uniform sampling systems, the corresponding distributed fusion filters are also given in [12-13]. However, the cross-covariance matrices are needed to obtain the fusion weights. Furthermore, the multi-rate fusion filters for systems with network constrains are studied in [14-16]. However, the state/measurement augmentation is not avoided.

In a recent study [17], by introducing a group of "dummy" random variables with Bernoulli distribution, the multi-rate fusion problem is transformed into an equivalent single rate fusion problem. The FFWM with smaller computational burden is proposed. However, the present fusion filter is only suitable for normal systems not for singular systems. Further, the parameter disturbance is not considered.

In this article, we study the filtering problem for multi-rate multi-sensor singular systems with parameter disturbance. Similar [17], the original multi-rate system is transformed into a single rate system with stochastic parameter by introducing random variables with Bernoulli distribution. Firstly, the LFs are proposed by projection theory. Then, the well-known weighting fusion algorithm by matrices is used to fuse all the LFs. The proposed LFs can reduce the computational cost since the state augmentation is avoided. Moreover, the proposed FFWM can give better performance than any LFs.

\section{Problem Formulation}

Consider the following linear discrete-time stochastic multi-rate singular systems measured by $Q$ sensors

$$
\begin{gathered}
A x(t b+b)=B x(t b)+C w(t b) \\
z_{r}\left(k b_{r}\right)=\left(D_{r}+\lambda_{r}\left(k b_{r}\right) \underline{D}_{r}\right) x\left(k b_{r}\right)+\underline{v}_{r}\left(k b_{r}\right), r \in\{1, \cdots, Q\}
\end{gathered}
$$

where $x(t b) \in \square^{n}$ is the state vector at $t b$ time instant, $z_{r}\left(k b_{r}\right) \in \square{ }^{n_{r}}, r \in\{1, \cdots, Q\}$ are the measured outputs at $k b_{r}$ time instant where $t$ denotes the $t$ th state updating point and $k$ denotes the $k$ th measurement sampling point. $A, B, C, D_{r}$ and $\underline{D}_{r} r \in\{1, \cdots, Q\}$ are known constant matrices. $w(t b) \in \square^{q}$ and $\underline{v}\left(k b_{r}\right) \in \square^{n_{r}}, r \in\{1, \cdots, Q\}$ are white noises. The state $x(t b)$ is updated at the highest rate with a period $b$ and the $r$ th sensor measurement $z_{r}\left(k b_{r}\right) \in \square{ }^{n_{r}}$ is sampled at a lower rate with a period $b_{r}=c_{r} b$ where $c_{r}$ is a positive integer. Multiplicative noises $\lambda_{r}\left(k b_{r}\right)$ are scalar white that are introduced to describe the structured perturbation in measurement matrices. They are of zero-mean with variance matrices $R_{r}^{\lambda}$. Also, zero mean of $\lambda_{r}\left(k b_{r}\right)$ means that parameter perturbations in both directions are equally likely. We assumed that $\lambda_{r}\left(k b_{r}\right)$ are mutually uncorrelated and are independent of $w\left(k b_{r}\right)$ and $\underline{v}\left(k b_{r}\right), r \in\{1, \cdots, Q\}$.

Assumption $1 A$ is a singular square matrix, i.e., $\operatorname{rank}(A)=n_{0}<n$. 
Assumption 2 System (1) is regular, i.e., $\operatorname{det}(s A-B) \neq 0, s$ is an arbitrary complex variable.

Assumption $3 w(t b)$ and $\underline{v}_{v}\left(k b_{r}\right)$ are correlated white noises with zero means and variance matrices $R^{w}, \underline{R}_{r}^{v}$ and cross-covariance matrices are $\mathrm{E}\left[w\left(k b_{r}\right) \underline{v}_{r}^{\mathrm{T}}\left(k b_{r}\right)\right]=\underline{S}_{r}, \mathrm{E}\left[v_{r}\left(k b_{r}\right) \underline{v}_{l}^{\mathrm{T}}\left(k b_{l}\right)\right]=$ $\underline{R}_{r l}^{v}, r, l \in\{1, \cdots, Q\}$, respectively.

Assumption 4 The initial state vector $x(0)$ is uncorrelated with $w(t b)$ and $\underline{v}_{(}\left(k b_{r}\right)$, and satisfies $\mathrm{E}\{x(0)\}=\sigma$ and $\mathrm{E}\left\{(x(0)-\sigma)(x(0)-\sigma)^{\mathrm{T}}\right\}=\Sigma$.

Our objective is to find the FFWM $\hat{x}_{o}(t b \mid t b)$ at the highest rate based on the measurement information $\left(z_{r}\left(k b_{r}\right), z_{r}\left(k b_{r}-b_{r}\right), \cdots, z_{r}(0)\right), r \in\{1, \cdots, Q\}$.

From Assumptions 1-2, there exist nonsingular matrices $M$ and $N$ [18], such that

$$
\begin{gathered}
M A N=\left[\begin{array}{ll}
A^{(1)} & 0 \\
A^{(2)} & 0
\end{array}\right], M B N=\left[\begin{array}{cc}
B^{(1)} & 0 \\
B^{(2)} & B^{(3)}
\end{array}\right], \\
M C=\left[\begin{array}{l}
C^{(1)} \\
C^{(2)}
\end{array}\right], D_{r} N=\left[\begin{array}{ll}
D_{r}^{(1)} & D_{r}^{(2)}
\end{array}\right], \quad \underline{D}_{r} N=\left[\begin{array}{ll}
\underline{D}_{r}^{(1)} & \underline{D}_{r}^{(2)}
\end{array}\right]
\end{gathered}
$$

where $A^{(1)}$ is a nonsingular lower-triangular matrix with the dimension $n^{(1)} \times n^{(1)}, B^{(1)}$ is a quasi lower-triangular matrix with the dimension $n^{(1)} \times n^{(1)}, B^{(3)}$ is a nonsingular lowertriangular matrix with the dimension $n^{(2)} \times n^{(2)}$. By introducing the transformation $x(t b)=N\left[x^{(1) \mathrm{T}}(t b), x^{(2) \mathrm{T}}(t b)\right]^{\mathrm{T}}$, where $x^{(1)}(t b) \in R^{n^{(1)}}, x^{(2)}(t b) \in R^{n^{(2)}}$, then systems (1) and (2) can be transformed into the following systems

$$
\left\{\begin{array}{c}
x^{(1)}(t b+b)=\bar{A} x^{(1)}(t b)+\bar{B}_{w}(t b) \\
z_{r}\left(k b_{r}\right)=\left(\bar{D}_{r}+\lambda_{r}\left(k b_{r}\right) \tilde{D}_{r}\right) x^{(1)}\left(k b_{r}\right)+\bar{v}_{r}\left(k b_{r}\right) \\
x^{(2)}(t b)=U x^{(1)}(t b)+R w(t b)
\end{array}\right.
$$

where $\bar{A}=\left(A^{(1)}\right)^{-1} B^{(1)} \quad, \quad \bar{B}=\left(A^{(1)}\right)^{-1} C^{(1)}, \quad \bar{D}_{r}=D_{r}^{(1)}+D_{r}^{(2)} U \quad, \quad \tilde{D}_{r}=\underline{D}_{r}^{(1)}+\underline{D}_{r}^{(2)} U$, $U=\left(B^{(3)}\right)^{-1} A^{(2)}\left(A^{(1)}\right)^{-1} B^{(1)}-\left(B^{(3)}\right)^{-1} B^{(2)}, \bar{v}_{r}\left(k b_{r}\right)=\left(T_{r}+\lambda_{r}\left(k b_{r}\right) \tilde{T}_{r}\right) w\left(k b_{r}\right)+\underline{v}_{r}\left(k b_{r}\right), \quad T_{r}=D_{r}^{(2)} R$, $\tilde{T}_{r}=\underline{D}_{r}^{(2)} R, R=\left(B^{(3)}\right)^{-1} A^{(2)}\left(A^{(1)}\right)^{-1} C^{(1)}-\left(B{ }^{(3)}\right)^{-} C^{(\vdots}$.

Also, we have the following statistical property

$$
\begin{gathered}
\mathrm{E}\left[w\left(k b_{r}\right) \bar{v}_{r}^{\mathrm{T}}\left(k b_{r}\right)\right]=R^{w} T_{r}^{\mathrm{T}}+\underline{S}_{r}=\bar{S}_{r}, \\
\mathrm{E}\left[\bar{v}_{r}\left(k b_{r}\right) \bar{v}_{r}^{\mathrm{T}}\left(k b_{r}\right)\right]=T_{r} R^{w} T_{r}^{\mathrm{T}}+T_{r} \underline{S}_{r}+\underline{S}_{r}^{\mathrm{T}} T_{r}^{\mathrm{T}}+\underline{R}_{r}^{v}+R_{r}^{\lambda} \tilde{T}_{r} R^{w} \tilde{T}_{r}^{\mathrm{T}}=\bar{R}_{r}^{v}, \\
\mathrm{E}\left[\bar{v}_{r}\left(k b_{r}\right) \bar{v}_{l}^{\mathrm{T}}\left(k b_{l}\right)\right]=T_{r} R^{w} T_{l}^{\mathrm{T}}+T_{r} \underline{S}_{l}+\underline{S}_{r}^{\mathrm{T}} T_{l}^{\mathrm{T}}+\underline{R}_{r l}^{v}=\bar{R}_{r l}^{v}
\end{gathered}
$$

Now, system (4) is transformed into the normal system with multiple sampling rates, multiple sensors and multiplicative noises. In the following section, we shall derive the distributed FFWM based on the weighting fusion algorithm in the linear minimum variance sense.

\section{Distributed FFWM}

Firstly, we transform the multi-rate fusion estimation problem into a single rate fusion estimation problem. Similar [17], we introduce white Bernoulli distributed variables $\xi_{r}(t b)$ with

$$
\xi_{r}(t b)= \begin{cases}1, & t b=k b_{r} \\ 0, & \text { else }\end{cases}
$$

Based on $\xi_{r}(t b)$, we can define the following variables 


$$
\begin{gathered}
y_{r}(t b)=\left\{\begin{array}{ll}
z_{r}\left(k b_{r}\right), & \xi_{r}(t b)=1 \\
0, & \text { else }
\end{array}, v_{r}(t b)=\left\{\begin{array}{ll}
\bar{v}_{r}\left(k b_{r}\right), & \xi_{r}(t b)=1 \\
0, & \text { else }
\end{array}, \gamma_{r}(t b)=\left\{\begin{array}{ll}
\lambda_{r}\left(k b_{r}\right), & \xi_{r}(t b)=1 \\
0, & \text { else }
\end{array},\right.\right.\right. \\
F_{r}(t b)=\xi_{r}(t b) \bar{D}_{r}, \tilde{F}_{r}(t b)=\xi_{r}(t b) \tilde{D}_{r}
\end{gathered}
$$

From the above definition, we see that $\xi_{r}(t b)=1$ denotes $y_{r}(t b)=z_{r}(t b)$ and $\xi_{r}(t b)=0$ denotes $y_{r}(t b)=0$. Then system (4) can be transformed into the following single rate multisensor system with multiplicative noise:

$$
\begin{gathered}
x^{(1)}(t b+b)=\bar{A} x^{(1)}(t b)+\bar{B} w(t b) \\
y_{r}(t b)=\left(F_{r}(t b)+\gamma_{r}(t b) \tilde{F}_{r}(t b)\right) x^{(1)}(t b)+v_{r}(t b)
\end{gathered}
$$

Next, we shall give the LFs $\hat{x}_{r}^{(1)}(t b \mid t b)$ based on the random variables $\left(\xi_{r}(t b), \xi_{r}(t b-b), \cdots, \xi_{r}(0)\right)$ and measurements $\left(y_{r}(t b), y_{r}(t b-b) \cdots, y_{r}(0)\right), r \in\{1, \cdots, Q\}$.

\subsection{Local filter}

Observe that systems (8) and (9) are transformed into the single rate systems. In the following, we will give the LFs $\hat{x}_{r}^{(1)}(t b \mid t b)$ and the corresponding estimation error variance matrices $P_{r}^{(1)}(t b \mid t b-b)$ for the $r$ th sensor subsystem by applying the classical Kalman filter.

Theorem 1 Under Assumptions 1-4, the LFs for systems (8) and (9) are computed by

$$
\begin{aligned}
& \hat{x}_{r}^{(1)}(t b \mid t b)=\hat{x}_{r}^{(1)}(t b \mid t b-b)+\xi_{r}(t b) K_{r}(t b) \varepsilon_{r}(t b) \\
& \hat{x}_{r}^{(1)}(t b+b \mid t b)=\bar{A} \hat{x}_{r}^{(1)}(t b \mid t b-b)+\xi_{r}(t b) L_{r}(t b) \varepsilon_{r}(t b) \\
& \varepsilon_{r}(t b)=y_{r}(t b)-F_{r}(t b) \hat{x}_{r}^{(1)}(t b \mid t b-b) \\
& K_{r}(t b)=P_{r}^{(1)}(t b \mid t b-b) F_{r}{ }^{\mathrm{T}}(t b) Q_{\varepsilon_{r}}^{-1}(t b) \\
& L_{r}(t b)=\left(\bar{A} P_{r}^{(1)}(t b \mid t b-b) F_{r}{ }^{\mathrm{T}}(t b)+\bar{B} \bar{S}_{r}\right) Q_{\varepsilon_{r}}^{-1}(t b) \\
& Q_{\varepsilon_{r}}(t b)=F_{r}(t b) P_{r}^{(1)}(t b \mid t b-b) F_{r}{ }^{\mathrm{T}}(t b)+\bar{R}_{r}^{v}+R_{r}^{\lambda} \tilde{F}_{r}(t b) X^{(1)}(t b) \tilde{F}_{r}{ }^{\mathrm{T}}(t b) \\
& X^{(1)}(t b+b)=\bar{A} X^{(1)}(t b) \bar{A}^{\mathrm{T}}+\bar{B} R^{w} \bar{B}^{\mathrm{T}} \\
& P_{r}^{(1)}(t b \mid t b)=P_{r}^{(1)}(t b \mid t b-b)-\xi_{r}(t b) K_{r}(t b) Q_{\varepsilon_{r}}(t b) K_{r}^{\mathrm{T}}(t b) \\
& P_{r}^{(1)}(t b+b \mid t b)=\left(\bar{A}-\xi_{r}(t b) L_{r}(t b) F_{r}(t b)\right) P_{r}^{(1)}(t b \mid t b-b) \\
& \times\left(\bar{A}-\xi_{r}(t b) L_{r}(t b) F_{r}\right)^{\mathrm{T}}+\left[\begin{array}{ll}
\bar{B} & -\xi_{r}(t b) L_{r}(t b)
\end{array}\right]\left[\begin{array}{ll}
R^{w} & \bar{S}_{r} \\
\bar{S}_{r}^{\mathrm{T}} & \bar{R}_{r}^{v}
\end{array}\right]\left[\begin{array}{ll}
\bar{B} & -\xi_{r}(t b) L_{r}(t b)
\end{array}\right]^{\mathrm{T}}
\end{aligned}
$$

where $\varepsilon_{r}(t b)$ is the innovation sequence with variance $Q_{\varepsilon_{r}}(t b), K_{r}(t b)$ is the filtering gain, $L_{r}(t b)$ is the one-step prediction gain, $X^{(1)}(t b)$ is the state second-order moment matrix, $P_{r}^{(1)}(t b \mid t b)$ is the filtering error variance matrix, $P_{r}^{(1)}(t b \mid t b-b)$ is the one-step prediction error variance matrix. The initial values are $\hat{x}_{r}^{(1)}(0 \mid-b)=\sigma, X_{r}^{(1)}(0)=\Sigma_{1}$ and $P_{r}^{(1)}(0 \mid-b)=\Sigma_{1}$ where $\Sigma_{1}$ is the first $n^{(1)} \times n^{(1)}$ block of $N^{-1}\left(\Sigma+\sigma \sigma^{\mathrm{T}}\right)\left(N^{-1}\right)^{\mathrm{T}}$.

Proof: This proof is analogous to [17].

Next, we shall derive $\hat{x}_{r}^{(2)}(t b \mid t b)$ based on $\hat{x}_{r}^{(1)}(t b \mid t b)$ and $\left(\xi_{r}(t b), \xi_{r}(t b-b), \cdots, \xi_{r}(0)\right)$.

Theorem 2. Under Assumptions 1-4, the LFs for system (5) are computed by

$$
\begin{gathered}
\hat{x}_{r}^{(2)}(t b \mid t b)=U \hat{x}_{r}^{(1)}(t b \mid t b)+R \hat{w}_{r}(t b \mid t b) \\
\hat{w}_{r}(t b \mid t b)=\xi_{r}(t b) \bar{S}_{r} Q_{\varepsilon_{r}}^{-1}(t b) \varepsilon_{r}(t b) \\
P_{r}^{(2)}(t b \mid t b)=G_{r}(t b) P_{r}^{(1)}(t b \mid t b-b) G_{r}^{\mathrm{T}}(t b)+H_{r}(t b)\left\lceil\begin{array}{ll}
R^{w} & \bar{S}_{r} \\
\bar{S}_{r}^{\mathrm{T}} & \bar{R}_{r}^{v}
\end{array} H_{r}^{\mathrm{T}}(t b)\right.
\end{gathered}
$$




$$
+\xi_{r}(t b) R_{r}^{\lambda}\left(U K_{r}(t b)+R \bar{S}_{r} Q_{\varepsilon_{r}}^{-1}(t b)\right) \tilde{F}_{r}(t b) X^{(1)}(t b) \tilde{F}_{r}^{\mathrm{T}}(t b)\left(U K_{r}(t b)+R{\overline{S_{r}}}_{r} Q_{\varepsilon_{r}}^{-1}(t b)\right)^{\mathrm{T}}
$$

where $G_{r}(t b)=U-\xi_{r}(t b)\left(U K_{r}(t b)+R \bar{S}_{r} Q_{s_{r}}^{-1}(t b)\right) F_{r}(t b), H_{r}(t b)=\left[\begin{array}{ll}R & -\xi_{r}(t b)\left(U K_{r}(t b)+R \bar{S}_{r} Q_{s_{r}}^{-1}(t b)\right)\end{array}\right]$, $\hat{w}_{r}(t b \mid t b)$ is the white noise filter, $P_{r}^{(2)}(t b \mid t b)$ is the filtering error variance matrix for $x^{(2)}(t b)$.

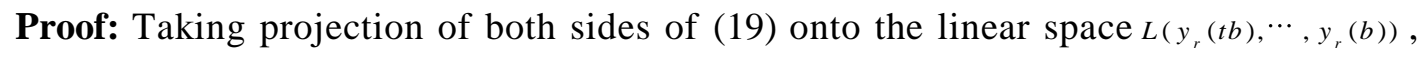
we have (19). The white noise filter $\hat{w}_{r}(t b \mid t b)$ is obtained by applying the projection theory [2]

$$
\hat{w}_{r}(t b \mid t b)=\hat{w}_{r}(t b \mid t b-b)+\mathrm{E}\left[w(t b) \varepsilon_{r}^{\mathrm{T}}(t b)\right] Q_{\varepsilon_{r}}^{-1}(t b) \varepsilon_{r}(t b)
$$

where the white noise one-step predictor $\hat{w}_{r}(t b \mid t b-b)$ is zero vector. Substituting (9) into (12) and using the definition of $\xi_{r}(t b)$, the innovation sequences can be rewritten as

$$
\varepsilon_{r}(t b)=\xi_{r}(t b) v_{r}(t b)+\gamma_{r}(t b) \tilde{F}_{r}(t b) x^{(1)}(t b)+F_{r}(t b) \tilde{x}_{r}^{(1)}(t b \mid t b-b)
$$

From $w(t b) \perp x^{(1)}(t b)$ and $w(t b) \perp \hat{x}_{r}^{(1)}(t b \mid t b-b)$, we have

$$
\mathrm{E}\left[w(t b) \varepsilon_{r}^{\mathrm{T}}(t b)\right]=\xi_{r}(t b) \mathrm{E}\left[w(t b) v_{r}^{\mathrm{T}}(t b)\right]=\xi_{r}(t b) \bar{S}_{r}
$$

Substituting (24) into (22), the (20) is obtained.

From (5) and (19), we have the filtering error equation for state $x^{(2)}(t b)$

$$
\tilde{x}_{r}^{(2)}(t b \mid t b)=U\left(x_{r}^{(1)}(t b)-\hat{x}_{r}^{(1)}(t b \mid t b)\right)+R\left(w(t b)-\hat{w}_{r}(t b \mid t b)\right)
$$

Substituting (10) and (20) into (25), and using $\left(\xi_{r}(t b)\right)^{2}=\xi_{r}(t b),(25)$ can be rewritten as

$$
\tilde{x}_{r}^{(2)}(t b \mid t b)=U \tilde{x}_{r}^{(1)}(t b \mid t b-b)+R w(t b)-\xi_{r}(t b)\left(U K_{r}(t b)+R \bar{S}_{r} Q_{\varepsilon_{r}}^{-1}(t b)\right) \varepsilon_{r}(t b)
$$

Substituting (23) into(26), the filtering error can be further rewritten as

$$
\begin{aligned}
& \tilde{x}_{r}^{(2)}(t b \mid t b)=G_{r}(t b) \tilde{x}_{r}^{(1)}(t b \mid t b-b)+H_{r}(t b)\left[w^{\mathrm{T}}(t b) \quad v_{r}^{\mathrm{T}}(t b)\right]^{\mathrm{T}} \\
& -\xi_{r}(t b) \gamma_{r}(t b)\left(U K_{r}(t b)+R \bar{S}_{r} Q_{\varepsilon_{r}}^{-1}(t b)\right) \tilde{F}_{r}(t b) x^{(1)}(t b)
\end{aligned}
$$

where $G_{r}(t b)$ and $H_{r}(t b)$ are defined as above. Substituting (27) into $P_{r}^{(2)}(t b \mid t b)=$ $\mathrm{E}\left[\tilde{x}_{r}^{(2)}(t b \mid t b) \tilde{x}_{r}^{(2) \mathrm{T}}(t b \mid t b)\right]$ and using $\mathrm{E}\left[\xi_{r}(t b) \gamma_{r}(t b)\right]=0$, we have $(21)$.

\subsection{Fusion filter}

Next, we will derive the cross-covariance matrices between any two LFs for the two reduced-order subsystems to obtain the fusion weights.

Theorem 3. For reduced-order subsystems (8)-(9) and subsystem (5), the filtering error cross-covariance matrices between the $r$ th and the $l$ th local filtering errors are computed by

$$
\begin{aligned}
& P_{r l}^{(1)}(t b \mid t b)=P_{r l}^{(1)}(t b \mid t b-b)-\xi_{l}(t b) P_{r l}^{(1)}(t b \mid t b-b) F_{l}{ }^{\mathrm{T}}(t b) K_{l}^{\mathrm{T}}(t b) \\
& -\xi_{r}(t b) K_{r}(t b) F_{r}(t b) P_{r l}^{(1)}(t b \mid t b-b)+\xi_{r}(t b) \xi_{l}(t b) K_{r}(t b) Q_{\varepsilon_{r l}}(t b) K_{l}{ }^{\mathrm{T}}(t b) \\
& P_{r l}^{(1)}(t b+b \mid t b)=\bar{A} P_{r l}^{(1)}(t b \mid t b-b) \bar{A}^{\mathrm{T}}+\bar{B} R^{w} \bar{B}^{\mathrm{T}} \\
& -\xi_{l}(t b) \bar{A} P_{r l}^{(1)}(t b \mid t b-b) F_{l}^{\mathrm{T}}(t b) L_{l}^{\mathrm{T}}(t b)-\xi_{r}(t b) L_{r}(t b) F_{r}(t b) P_{r l}^{(1)}(t b \mid t b-b) \bar{A}^{\mathrm{T}} \\
& +\xi_{r}(t b) \xi_{l}(t b) L_{r}(t b) F_{r}(t b) P_{r l}^{(1)}(t b \mid t b-b) F_{l}^{\mathrm{T}}(t b) L_{l}^{\mathrm{T}}(t b) \\
& -\xi_{r}(t b) L_{r}(t b) \bar{S}_{r}^{\mathrm{T}} \bar{B}-\xi_{l}(t b) \bar{B} \bar{S}_{l} L_{l}^{\mathrm{T}}(t b)+\xi_{r}(t b) \xi_{l}(t b) L_{r}(t b) \bar{R}_{r l}^{v} L_{l}^{\mathrm{T}}(t b) \\
& Q_{\varepsilon_{r l}}(t b)=F_{r}(t b) P_{r l}^{(1)}(t b \mid t b-b) F_{l}{ }^{\mathrm{T}}(t b)+\bar{R}_{r l}^{v} \\
& P_{r l}^{(2)}(t b \mid t b)=G_{r}(t b) P_{r l}{ }^{(1)}(t b \mid t b-b) G_{l}{ }^{\mathrm{T}}(t b)+H_{r}(t b) \mid\left[\begin{array}{ll}
R^{w} & \left.\overline{S_{l}}\right\rceil \\
\overline{S_{r}} & \overline{R_{r l}}
\end{array}\right] H_{l}{ }^{\mathrm{T}}(t b) \\
& P_{r l}^{(12)}(t b \mid t b)=\left(I_{n^{(1)}}-\xi_{r}(t b) K_{r}(t b) F_{r}(t b)\right) P_{r l}^{(1)}(t b \mid t b-b) G_{l}^{\mathrm{T}}(t b)-\xi_{r}(t b) K_{r}(t b)\left[\bar{S}_{r}^{\mathrm{T}} \quad \bar{R}_{r l}^{v}\right] H_{l}^{\mathrm{T}}(t b)
\end{aligned}
$$


where $Q_{\varepsilon_{u}}(t b)$ is the innovation variance cross-covariance matrices. The initial values are $P_{r l}^{(1)}(0 \mid-b)=\Sigma_{1}$.

Proof: From [17], we have (28)-(30). Substituting (27) into $P_{r l}^{(2)}(t b \mid t b)=\mathrm{E}\left[\tilde{x}_{r}^{(2)}(t b \mid t b) \tilde{x}_{l}^{(2) \mathrm{T}}(t b \mid t b)\right]$, we have (31). Subtracting (10) from (8), we have the filtering error equation of state $x^{(1)}(t b \mid t b)$

$$
\begin{aligned}
& \tilde{x}_{r}^{(1)}(t b \mid t b)=\left(I_{n^{(1)}}-\xi_{r}(t b) K_{r}(t b) F_{r}(t b)\right) \tilde{x}_{r}^{(1)}(t b \mid t b-b) \\
& \quad-\xi_{r}(t b) K_{r}(t b) v_{r}(t b)-\xi_{r}(t b) \gamma_{r}(t b) K_{r}(t b) \tilde{F}_{r}(t b) x^{(1)}(t b)
\end{aligned}
$$

Substituting (33) and (27) into $P_{r l}^{(12)}(t b \mid t b)=\mathrm{E}\left[\tilde{x}_{r}^{(1)}(t b \mid t b) \tilde{x}_{l}^{(2) \mathrm{T}}(t b \mid t b)\right]$, we have(32).

Next, we shall give the following optimal FFWMs $\hat{x}_{o}^{(q)}(t b \mid t b), q \in\{1,2\}$ by using LFs and corresponding filtering error variance matrices and cross-covariance matrices

$$
\hat{x}_{o}^{(q)}(t b \mid t b)=P_{o}^{(q)}(t b \mid t b) \Xi^{(q) \mathrm{T}} \Omega^{(q)-1}(t b)\left[\hat{x}_{1}^{(q) \mathrm{T}}(t b \mid t b), \hat{x}_{2}^{(q) \mathrm{T}}(t b \mid t b), \cdots, \hat{x}_{Q}^{(q) \mathrm{T}}(t b \mid t b)\right]^{\mathrm{T}}, q \in\{1,2\}
$$

where $\Xi^{(q)}=\left[I_{n^{(q)}}, \cdots, I_{n^{(q)}}\right]^{\mathrm{T}}$ are constant matrices with the dimensions $n^{(q)} Q \times n^{(q)}$. $\Omega^{(q)}(t b)=\left(P_{r l}^{(q)}(t b \mid t b)\right)_{n^{(q)} Q \times n^{(q)} Q}, r, l \in\{1, \cdots, Q\}$ are the matrices with the dimensions $n^{(q)} Q \times n^{(q)} Q$. $P_{o}^{(a)}(t b \mid t b), q \in\{1,2\}$ are the corresponding fusion filtering error variance and computed by

$$
P_{o}^{(q)}(t b \mid t b)=\left(\Xi^{(q) \mathrm{T}} \Omega^{(q)-1}(t b) \Xi^{(q)}\right)^{-1}, \quad q \in\{1,2\}
$$

where $P_{r l}^{(1)}(t b \mid t b)$ and $P_{r l}^{(2)}(t b \mid t b)$ are given by Theorems 1-3, Further we have $P_{o}^{(q)}(t b \mid t b) \leq P_{r}^{(q)}(t b \mid t b), r \in\{1, \cdots, Q\}, q \in\{1,2\}$.

Theorem 4 For the original multi-rate singular system (1)-(3), we have the following FFWM

$$
\hat{x}_{o}(t b \mid t b)=N\left[\hat{x}_{o}^{(1) \mathrm{T}}(t b \mid t b) \quad \hat{x}_{o}^{(2) \mathrm{T}}(t b \mid t b)\right]^{\mathrm{T}}
$$

The corresponding fusion filtering error variance matrix is computed by

$$
P_{o}(t b \mid t b)=N\left[\begin{array}{ll}
P_{o}^{(1)}(t b \mid t b) & P_{o}^{(12)}(t b \mid t b) \\
P_{o}^{(21)}(t b \mid t b) & P_{o}^{(2)}(t b \mid t b)
\end{array}\right] N^{\mathrm{T}}
$$

where $P_{o}^{(12)}(t b \mid t b)$ is the cross-covariance matrix between the two reduced order subsystems and is computed by

$$
P_{o}^{(12)}(t b \mid t b)=P_{o}^{(1)}(t b \mid t b) \Xi^{(1) \mathrm{T}} \Omega^{(1)-1}(t b) \Omega^{(12)-1}(t b) \Omega^{(2)-1}(t b) \Xi^{(2)} P_{o}^{(2)}(t b \mid t b)
$$

where $\Omega^{(12)}(t b)=\left(P_{r l}^{(12)}(t b \mid t b)\right)_{n^{(1)} Q \times n^{(2)} Q}, P_{o}^{(21)}(t b \mid t b)=P_{o}^{(12) \mathrm{T}}(t b \mid t b) . P_{o}^{(1)}(t b \mid t b)$ and $P_{o}^{(2)}(t b \mid t b)$ is computed by (35), $P_{r l}^{(12)}(t b \mid t b)$ is given by Theorem 3 .

\section{Simulation results}

Consider the multi-rate system (1)-(2) measured by two sensors, where $M=N=I_{4}$,

$$
\begin{aligned}
& A^{(1)}=\left[\begin{array}{cc}
-2.13 & 0 \\
1 & 0.5
\end{array}\right], A^{(2)}=\left[\begin{array}{cc}
1 & 0.5 \\
0 & -1
\end{array}\right], B^{(1)}=\left[\begin{array}{cc}
-1 & 0.2 \\
-0.5 & 0
\end{array}\right], B^{(2)}=\left[\begin{array}{cc}
1 & -0.5 \\
0 & -1
\end{array}\right], B^{(3)}=\left[\begin{array}{cc}
-0.5 & 0 \\
-1 & 2
\end{array}\right], \\
& C^{(1)}=\left[\begin{array}{cc}
0.5 & 0 \\
0 & 0.8
\end{array}\right], C^{(2)}=\left[\begin{array}{cc}
0.8 & 0 \\
0 & -0.6
\end{array}\right], D_{1}^{(1)}=\left[\begin{array}{cc}
1 & 0.5 \\
0 & 1
\end{array}\right], D_{1}^{(2)}=\left[\begin{array}{cc}
1 & 0 \\
0 & 1
\end{array}\right], D_{2}^{(1)}=\left[\begin{array}{cc}
1 & 0 \\
0 & 0.8
\end{array}\right], D_{2}^{(2)}=\left[\begin{array}{cc}
0.5 & 1 \\
1 & 0.6
\end{array}\right], \\
& \underline{D}_{-1}^{(1)}=\left[\begin{array}{cc}
0.1 & 0 \\
0 & 0.1
\end{array}\right], \underline{D}_{1}^{(2)}=\left[\begin{array}{cc}
0.1 & 0 \\
0 & 0.2
\end{array}\right], \underline{D}_{2}^{(1)}=\left[\begin{array}{cc}
0.05 & 0 \\
0 & 0.08
\end{array}\right], \underline{D}_{2}^{(2)}=\left[\begin{array}{cc}
0 & 0.1 \\
0.1 & 0
\end{array}\right] . \text { The system noise } w(t b) \text { and }
\end{aligned}
$$
observation noises $\underline{v}\left(k b_{r}\right), b_{r}=c_{r} b, r=1,2$ are white noises with mean-zero, variances $R^{w}$, 
$R_{r}^{v}$. Our aim is to find the distributed optimal FFWM $\hat{x}_{o}(t b \mid t b)$. In simulation, we set $c_{1}=2$, $c_{2}=3, R^{w}=I_{2}, R_{1}^{v}=4 I_{2}, R_{2}^{v}=2 I_{2}, x^{(1)}(0)=\left[\begin{array}{ll}0 & 0\end{array}\right]^{\mathrm{T}}$ and $P_{01}=0.1 I_{2}$.
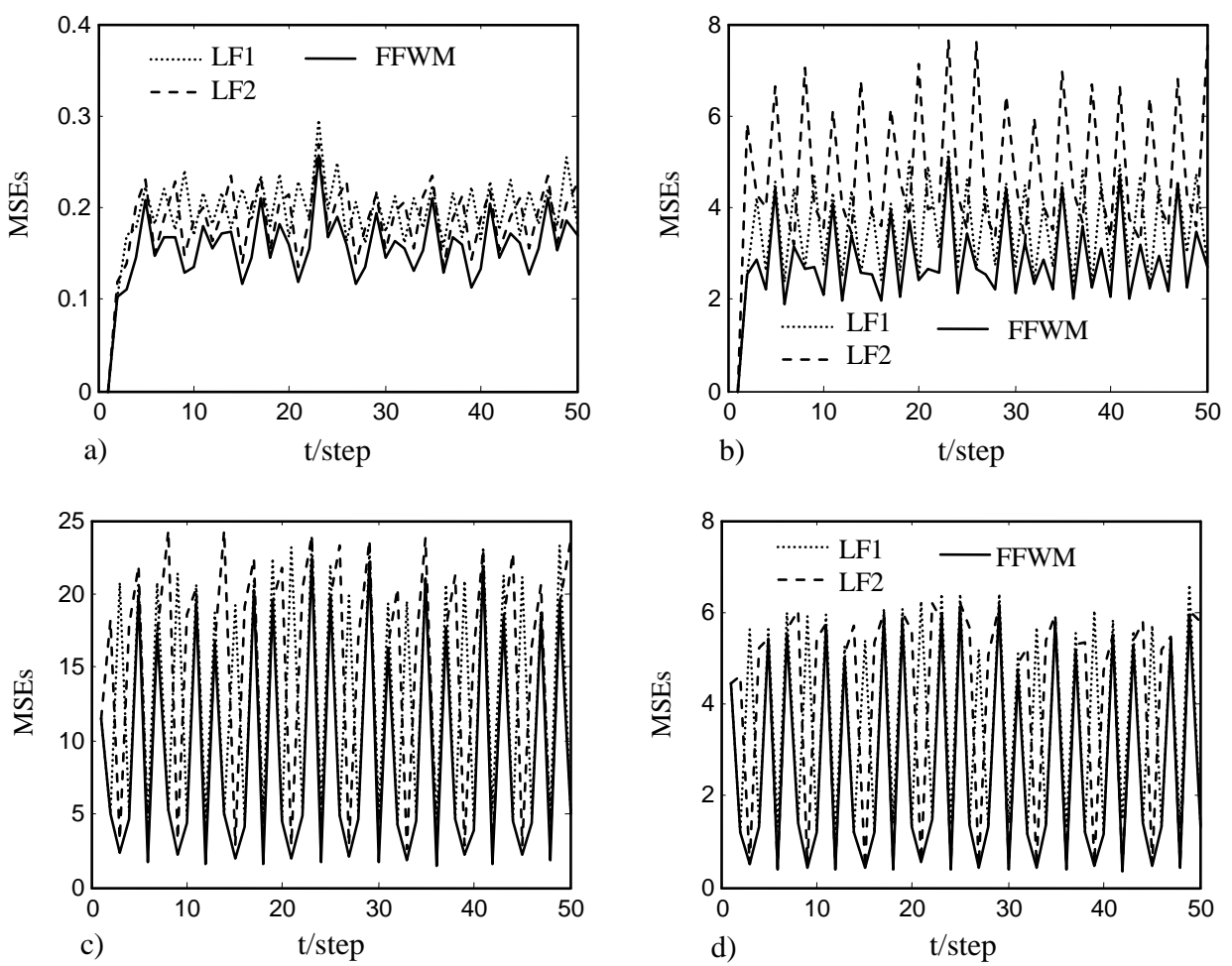

Figure 1. Comparison Curves of MSEs of FFWM and All LFs

The comparison curves of the mean square error (MSE) of the FFWM and all LFs by 200 times Monte Carlo tests are given in Figure 1. From Figure 1, we see that the proposed FFWM has the higher accuracy than any LFs.

\section{Conclusion}

In this paper a multi-rate multi-sensor distributed information fusion filtering problem for linear stochastic singular system with measurement multiplicative noise is studied. The LFs and corresponding filtering error variance matrices for the two reduced-order subsystems are derived. Furthermore, the FFWM for the original multi-rate stochastic singular system is obtained. Simulation results show better performance than any LFs.

\section{Acknowledgements}

This work was supported by Foundation of Heilongjiang Education Committee (Nos. 12541632 and 12531159), by Natural Science Foundation of Heilongjiang Province (No. F201426), and by Harbin University of Commerce Youth Fund.

\section{References}

[1] Q. Pan, Z. F. Wang, Y. Liang, F. Yang and Z. G. Liu, "Basic Methods and Progress of Information Fusion (II)", Control Theory \& Applications, vol. 29, no. 10, (2012), pp. 1233-1244.

[2] B. D. O. Anderson and J. B. Moore, "Optimal Filtering, Englewood Cliffs, New Jersey", Prentice-Hall (1979). 
[3] N. A. Carlson, "Federated Square Root Filter for Decentralized Parallel Processes", IEEE Trans. on Aerospace and Electronic Systems, vol. 26, no. 3, (1990), pp. 517-525.

[4] K. H. Kim, "Development of Track to Track Fusion Algorithm", Proceedings of the American Control Conference, (1994), pp. 1037-1041, Maryland.

[5] S. L. Sun and Z. L. Deng, "Multi-sensor Optimal Information Fusion Kalman Filte", Automatica, vol. 40, no. 6, (2004), pp. 1017-1023.

[6] F. Ding and T. W. Chen, "Modeling and Identification of Multirate Systems", Acta Automatica Sinica, vol. 31, no. 1, (2005), pp. 105-122.

[7] B. S. Chen, C. W. Lin and Y. L. Chen, "Optimal Signal Reconstruction in Noisy Filter Bank Systems", Multirate Kalman Synthesis Filtering Approach, IEEE Trans. on Signal Processing, vol. 43, no. 11, (1995), pp. 2496-2504.

[8] J. Wang, C. Z. Han and X. R. Li, “Asynchronous Multisensor Data Fusion”, Control And Decision, vol. 17, no. 6, (2001), pp. 877-881.

[9] Q. B. Ge, G. A. Wang, T. H. Tang and C. L. Wen, "The Research on Asynchronous Data Fusion Algorithm Based on Sampling of Rational Number Times", Acta Electronica Sinica, vol. 34, no. 3, (2006), pp. 544-548.

[10] A. B. Qiu, C. L. Wen and B. Jiang, "Optimal State Fusion Estimate Based on Asynchronous Multisensor Sampled Measurements", Acta Electronica Sinica, vol. 38, no. 7, (2010), pp. 1483-1488.

[11] J. Ma, H. L. Lin, and S. L. Sun, "Distributed Fusion Filter for Asynchronous Multi-rate Multi-sensor Nonuniform Sampling Systems", Proceedings of the 15th International Conference on Information Fusion, (2012), pp. 1645-1652, Singapore.

[12] Y. L. Liu, L. P. Yan, Y. Q. Xia, M. Y. Fu, and B. Xiao, "Multirate Multisensor Distributed Data Fusion Algorithm for State Estimation with Cross-Correlated Noises", Proceedings of the 32nd Chinese Control Conference, (2013), pp. 4682-4687, Xi'an.

[13] F. F. Peng and S. L. Sun, "Distributed Fusion Estimation for Multi-sensor Multi-rate Systems with Stochastic Observation Multiplicative Noises", Mathematical Problems in Engineering, Article ID 373270, 8 pages, (2014).

[14] J. Ma and S. L. Sun, "Distributed Fusion Filter for Multi-rate Multi-sensor Systems with Packet Dropouts", Proceedings of the 10th World Congress on Intelligent Control and Automation, (2012), pp. 4502-4506, Beijing.

[15] M. S. Mahmoud and M. F. Emzir, "State Estimation with Asynchronous Multi-rate Multi-smart Sensors", Information Sciences, (2012), pp. 196: 15-27.

[16] W. A. Zhang, G. Feng and L.Yu, "Multi-rate Distributed Fusion Estimation for Sensor Networks with Packet Losses", Automatica, vol. 48, no. 9), (2012), pp. 2016-2028.

[17] J. Ma, S. L. Sun, "Distributed Fusion Filter for Multi-rate Multi-sensor Systems with Packet Dropouts", In Proceedings of the 10th World Congress on Intelligent Control and Automation, (2012), pp. 4502-4506, Beijing.

[18] S. L. Sun, J. Ma, "Distributed Reduced-order Optimal Fusion Kalman Filters for Stochastic Singular Systems”, Acta Automatica Sinica, vol. 32, no. 2, (2006), pp. 285-290.

\section{Authors}

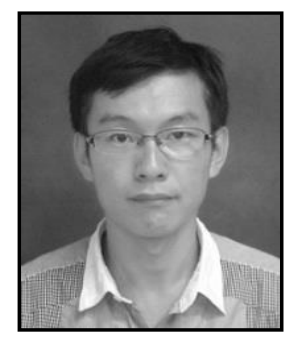

Hao Jin, he was born in Heilongjiang, China, in 1981. He received the B.E. and M.E. degrees from Heilongjiang University, Harbin, China, in 2004 and 2007, respectively. His current research interests include information fusion filtering and image processing.

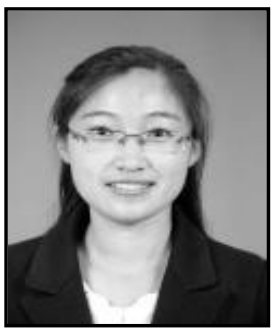

Jing Ma, she was born in Heilongjiang, China, in 1979. She received the M.E. and Ph.D. degrees from Heilongjiang University, Harbin, China, in 2007 and 2012, respectively. Her current research interests include sensor network and information fusion filtering. 


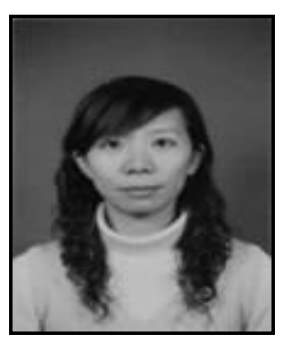

Yun Li, she was born in Heilongjiang, China, in 1978. She received the B.E. and M.E. degrees from Heilongjiang University, Harbin, China, in 2001 and 2005, respectively. Her major researches are state estimation, information fusion, etc.

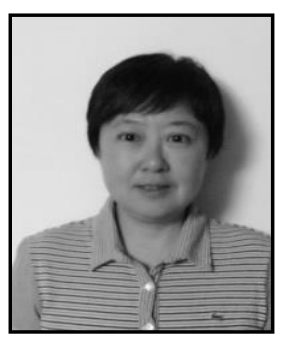

Ming Zhao, she was born in Heilongjiang, China, in 1965. She received the B.E. and M.E. degrees from Harbin Engineering University, Harbin, China, in 1987 and 1990, respectively. Her current research interests include sensor network and image processing. 
International Journal of Multimedia and Ubiquitous Engineering Vol. 10, No. 2 (2015) 